\title{
ScienceCentral: open access full-text archive of scientific journals based on Journal Article Tag Suite regardless of their languages
}

Sun Huh

Department of Parasitology and Institute of Medical Education, College of Medicine, Hallym University, Chuncheon, Korea

Corresponding author: shuh@hallym.ac.kr

\begin{abstract}
ScienceCentral, a free or open access, full-text archive of scientific journal literature at the Korean Federation of Science and Technology Societies, was under test in September 2013. Since it is a Journal Article Tag Suite-based full text database, extensible markup language files of all languages can be presented, according to Unicode Transformation Format 8-bit encoding. It is comparable to PubMed Central: however, there are two distinct differences. First, its scope comprises all science fields; second, it accepts all language journals. Launching ScienceCentral is the first step for free access or open access academic scientific journals of all languages to leap to the world, including scientific journals from Croatia.
\end{abstract}

Key words: periodicals as topic; language; database; science

Accepted: September 15, 2013

It is possible to trace 56 biomedical journals from Croatia, referenced in the NCBI databases. Twelve of these journals are currently indexed in Medline. Out of 12 Medline journals, only Biochemia Medica and the Croatian Medical Journal are in English and the other ten journals are in Croatian and in English. There are also three journals in English not currently indexed in Medline: Kinesiology, Neurologia Croatica and Psihoterapija (1). Only the Croatian Medical Journal has been indexed in PubMed Central (PMC) from the issue of volume 47 (2006). Twelve Medline journals are easily accessible through PubMed; meanwhile, other journals not currently indexed in Medline have difficulty broadening their visibility to most biomedical researchers. Although Google Scholar is a very powerful literature searching tool, the impact of PubMed is still overwhelming in the field of biomedicine. It is the reason why many biomedical journal editors want to add their journals in PubMed.

There are two ways to be included in PubMed: one is to be Medline journal through review of journal title and assessment of the quality of its content by Literature Selection Technical Review Committee; the other is to become a PubMed Central journal. The former process is not easy to pass these days; however, the latter is easy if the journal is written only in English and the PMC extensible markup language $(\mathrm{XML})$ file meets the technical criteria. For example, more than 60 biomedical English journals from Korea, not indexed in Medline, began to make PMC XML files and to submit them to PMC. Three journals in English, mentioned above, can be candidates of PMC, ultimately to be included in PubMed. One method of definite merit, to become a PMC journal, is visibility via Pubreader, a new reader-friendly way to look at articles. It is designed for enhancing readability on tablet and other small screen devices, as well as web browsers of desktops or laptops. It is the reason why Biochemia Medica wants to become a PMC journal. It already passed the first process of evaluation. When a PMC XML file is dispatched to the PMC, it will be included in PMC after technical confirmation.

As for Croatian language journals not indexed in Medline, what can their editors do for better visibility to world physicians and researchers? The answer is ScienceCentral; a free or open access fulltext archive of scientific journal literature at the Korean Federation of Science and Technology Societies, under test in September 2013. (2). Since ScienceCentral is a Journal Article Tag Suite (JATS)- 
based full text database, it accepts all languages, according to Unicode Transformation Format 8-bit encoding (3). It accepts journals if abstract, figures, tables and references are written in English. There are two most distinct differences with PMC: first, ScienceCentral's scope is all science fields; second, ScienceCentral accepts all language journals in the world. Other maintenance models are almost the same as PMC, which is supported by the Government's budget. Biochemia Medica is already included in ScienceCentral. Any editors who are interested in ScienceCentral, can submit JATS XML files to ScienceCentral.

In the market of world scholarly journals, the influence of large, international, commercial printing companies is tremendous. They lead the international standard of scholarly journals, such as Digital Object Identifier (DOI). Half of scholarly journals are produced by the top 5 commercial printing companies; the top 5 commercial companies share $45.6 \%$ of all papers; the top 5 commercial companies receive $41.8 \%$ of all citations from Web of Science (4). A portion of scholarly journals published by academic societies is about $30 \%$. To compete with commercial printing companies, and to survive in the journal market, society journals should be able to catch up with new standards or new advancements of information technologies applicable to journals. These are JATS XML, Pubreader, DOI, CrossCheck, CrossMark (5), FundRef (6), Open Researcher and Contributor ID (ORCID) (7), QR code, mobile applications and multimedia. Owing to the advancement of programming, the realisation of those technologies is simple and easy. The application of those information technologies can be seen in the Journal of Educational Evaluation for Health Professions (8). Furthermore, society journals should be included in free or open access full text database platforms, comparable to those of commercial printing companies: examples are PMC, KoreaMed Synapse (9), and ScienceCentral. The visibility and the citation of the journals included may increase through those platforms.

ScienceCentral can be a good platform for free or open access journals of all languages and all science fields. Since it is based on JATS, other technical support for Pubreader, CrossMark, FundRef, ORCID, and multimedia is possible. Each journal can use ScienceCentral as a landing page of DOI and also each paper can be hyperlinked from a journal homepage.
English as a language of science is already wellknown; however, production of full text XML is another survival strategy whereby scientific description can exist in languages other than English. Translation of local or regional languages to another is still not complete. Technical advancement will erode the language barrier, year by year. In the near future, researchers will be able to comprehend other language papers without difficulty if abstract, tables, figures and references are in English, and the main text is not written in English. The launching of ScienceCentral is the first step for academic scientific journals of all languages to leap into the world. This new project has the complete support of the Korean Federation of Science and Technology Societies, funded by the Government of the Republic of Korea. Let us provide invaluable scientific information to all scientists in the world through ScienceCentral.

\section{Acknowledgments}

This work was supported by the Korean Federation of Science and Technology Societies Grant funded by the Korean Government (2013).

\section{Potential conflict of interest}

None declared.

\section{References}

1. NLM Catalog: Journals referenced in the NCBI Databases. Bethesda: U.S. National Institute of Health; 2013. Available at: http://www.ncbi.nlm.nih.gov/nlmcatalog/journals/. Accessed September 10, 2013.

2. ScienceCentral. Seoul (KR): Korean Federation of Science and Technology Societies; 2013. Available at: http://e-sciencecentral.org/. Accessed September 10, 2013.

3. Journal Article Tag Suite. Bethesda: U.S. National Institute of Health; 2013. Available at: http://jats.n/m.nih.gov/. Accessed September 10, 2013.

4. Didegah F, Gazni A. The extent of concentration in journal publishing. Learn Publ 2011;24:303-10. http://dx.doi. org/10.1087/20110408.

5. CrossMark. Oxford (UK): CrossRef; 2013. Available at: http:// www.crossref.org/crossmark/. Accessed September 10, 2013.

6. FundRef. Oxford (UK): CrossRef; 2013. Available at: http:// wWw.crossref.org/fundref/. Accessed September 10, 2013.

7. ORCID. Bethesda, MD: ORCID; 2013. Available at: $h t t p: / / o r-$ cid.org/. Accessed September 10, 2013.

8. Huh S. Revision of the instructions to authors to require a structured abstract, digital object identifier of each reference, and author's voice recording may increase journal access. J Educ Eval Health Prof 2013;10:3. http://dx.doi. org/10.3352/jeehp.2013.10.3.

9. Huh S. Medical databases from Korea and abroad. J Korean Med Assoc 2010;53:659-67. http://dx.doi.org/10.5124/ jkma.2010.53.8.659. 